\title{
A GESTÃO POR PROCESSOS GERA MELHORIA DE QUALIDADE E REDUÇÃO DE CUSTOS: O CASO DA UNIDADE DE ORTOPEDIA E TRAUMATOLOGIA DO HOSPITAL DAS CLÍNICAS DA UNIVERSIDADE FEDERAL DE PERNAMBUCO
}

\author{
PROCESS MANAGEMENT IMPROVES QUALITY AND REDUCES COSTS: \\ THE CASE OF THE ORTHOPEDICS AND TRAUMA UNIT AT THE HOSPITAL \\ DAS CLÍNICAS AT THE FEDERAL UNIVERSITY OF PERNAMBUCO
}

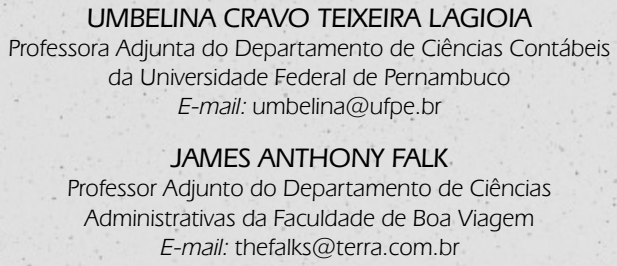

UMBELINA CRAVO TEIXEIRA LAGIOIA

Professora Adjunta do Departamento de Ciências Contábeis da Universidade Federal de Pernambuco

E-mail: umbelina@ufpe.br

JAMES ANTHONY FALK

Professor Adjunto do Departamento de Ciências

Administrativas da Faculdade de Boa Viagem E-mail: thefalks@terra.com.br

\author{
JOSÉ FRANCISCO RIBEIRO FILHO \\ Professor Adjunto do Departamento de Ciências Contábeis \\ da Universidade Federal de Pernambuco \\ E-mail: francisco.ribeiro@ufpe.br \\ JERONYMO JOSÉ LIBONATI \\ Professor Adjunto do Departamento de Ciências \\ Contábeis da Universidade Federal de Pernambuco \\ E-mail:jeronymolibonati@yahoo.com.br
}

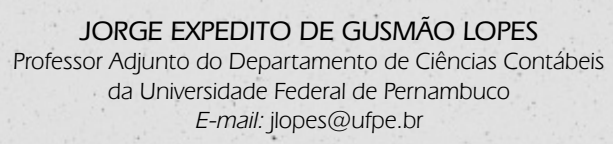

\section{RESUMO}

Este artigo teve como objetivo principal verificar até que ponto a utilização de protocolos de atendimento médicohospitalar pode promover um incremento na qualidade dos serviços prestados via padronização de processos, no mesmo tempo em que propicia umá redução nos custos diretamente relacionados aos procedimentos protocolados. O estudo em tela ocorreu na Unidade de Ortopedia e Traumatologia do Hospital das Clínicas da Universidade Federal de Pernambuco, e se refere ao procedimento de artroplastia de joelho ou colocação de prótese no joelho. Dados foram coletados durante os anos de 2004 e 2005 sobre indicadores de qualidade e sobre os custos diretos da cirurgia e da internação referentes a 89 pacientes que se submeteram ao procedimento sob estudo. A pesquisa foi realizada em dois momentos distintos: antes e após a implantação do protocolo que envolveu todo o processo médicohospitalar desde o seu atendimento inicial no ambulatório até sua alta do hospital. Por meio da análise dos dados ficou evidenciado que o protocolo implantado promoveu uma redução no tempo médio de espera para a realização da cirurgia da ordem de 3 dias, permitiu a diminuição de 7 dias na permanência total dos pacientes, reduziu em cerca de 20 minutos o tempo médio de duração da cirurgia. Todavia, não se conseguiu reduzir de maneira significativa os custos diretos de consumo, mas proporcionou uma queda em todos os custos diretos vinculadas à internação.

Palavras-chave: Indicadores de qualidade hospitalar. Protocolos médicos. Hospitais universitários.

\section{ABSTRACT}

This paper aims to verify to which extent the use of a medical-hospital treatment protocol can promote quality improvement in the services provided by means of process standardization, while at the same time reducing surgical and inpatient costs directly related to the procedure under protocol. The study took place at the Orthopedics Department of the Hospital das Clínicas at the Federal University of Pernambuco and referred to a knee arthroplasty or prosthetic knee replacement procedure. Data was collected during the period from 2004 to 2005 regarding quality indicators and direct surgical and inpatient costs for 89 patients submitted to the procedure under observation. The study looked at two distinct time periods: before and after the implementation of the protocol that involved the entire medical and administrative processes, ranging from the initial contact of the patient at the outpatient service up to the surgery and discharge from the hospital ward. Statistical data analysis made it evident that the protocol implemented at the hospital promoted a reduction in the mean waiting period of three days from time of admission to surgery, of seven days in the overall length of the patient' stay, a mean reduction of 20 minutes in the time of surgery, although no significant cost reduction in the materials consumed during the surgery itself was observed. It also proportioned a reduction in all direct cost categories related to the inpatient period.

Keywords: Hospital quality indicators. Medical protocols. University hospitals. 


\section{INTRODUÇÃO}

\subsection{A busca pela qualidade e pelo controle dos custos na assistência médico-hospitalar brasileira}

Desde algum tempo, uma verdadeira revolução administrativa vem ocorrendo nos hospitais brasileiros. Suas estruturas, antes voltadas para atender as exigências médicas, passam agora a se direcionar para a forma como seus serviços são prestados, cuidando de detalhes anteriormente não considerados importantes (ANDRADE, 2000).

Uma das razões que levou a essa mudança de postura decorre de uma visível transformação por parte dos principais agentes financiadores do sistema. Segundo Beulke e Bertó (1997), o mercado hospitalar brasileiro pode ser dividido em três grandes segmentos:

a) pacientes conveniados pelo SUS;

b) pacientes particulares;

c) pacientes com convênios privados.

As receitas oriundas da prestação de serviços via SUS não permitem, na maioria dos casos, a obtenção de grandes resultados por parte das instituições. O paciente particular, cuja receita normalmente apresenta um resultado positivo, é um segmento em gradativa extinção. Os convênios privados, por sua vez, vêm há um certo tempo procurando se estruturar de forma técnica e consistente diante dos termos preço e custo de atendimento (grifo dos autores). E é justamente através do confronto dessas duas variáveis que surge o primeiro grande problema sentido de igual maneira pelos três segmentos citados: como estabelecer um valor ou um preço para cada serviço prestado?

Rutledge (1996) complementa esse raciocínio ao afirmar que o meio ambiente hospitalar vem progressivamente demandando um maior entrosamento entre os médicos e os administradores dessas instituições no que diz respeito ao controle dos custos e à melhoria da qualidade. $\mathrm{Na}$ opinião dessa autora, apesar do corpo médico exercer um papel fundamental dentro dos hospitais e de responder por aproximadamente $75 \%$ de todos os custos da instituição, ele raramente é consultado ou informado sobre o planejamento financeiro e sobre as tomadas de decisões realizadas pelos administradores. Rutledge (1996) entende que esse comportamento deve ser revertido em nome da "boa saúde" da instituição. Em suas pesquisas ela verificou que o envolvimento da equipe médica na tomada de decisões administrativas promoveu uma redução dos custos e uma melhoria de desempenho nas atividades desenvolvidas no hospital como um todo.

Na opinião de Horn, Sharkey e Limmasch (1997), um dos principais desafios a ser enfrentado no meio ambiente hospitalar pode ser sintetizado da seguinte forma: como controlar os custos e ao mesmo tempo manter uma boa qualidade no padrão de atendimento?

A resposta a essa pergunta, de acordo com Nogueira (2003), passa pela padronização de processos. No entanto, algumas questões surgem com freqüência quando se começa a discutir sobre a padronização na área da saúde. A primeira delas é: como padronizar uma área em que cada paciente é único e na qual cada quadro clínico é absolutamente peculiar? A segunda questão freqüente é: não seria a padronização uma forma massificante de nivelar as pessoas, engessá-las e tolhê-las na forma de trabalhar?

Bittar (2000) entende os processos na área da saúde guardam entre si aspectos relacionados à diversidade, complexidade e intersetoriedade. Nesse sentido, ele afirma que:

- para um mesmo processo, em diferentes pacientes, pode-se necessitar de medicamentos com diferentes dosagens, assim como uma mesma doença admite diferentes tratamentos, invasivos, não-invasivos ou simplesmente a observação;

- uma mesma doença pode exigir um número maior e diferentes exames complementares para ser esclarecida;

- a coordenação de uma equipe multiprofissional em saúde pode ser dificultada pelas diferentes categorias profissionais que a compõem, bem como pela diversidade de formação que cada faculdade imprime, desenvolvendo processos de maneiras e custos diferentes, embora corretos.

Em reposta a esses posicionamentos, explica-se que na realidade o que se padroniza são os processos que serão utilizados ou não, em função da peculiaridade de cada paciente. A padronização, da forma como preconizada na gestão pela qualidade, estimula a criatividade e a participação de todos, trazendo no seu bojo o conceito de melhoria contínua: um bom padrão é aquele que sofre melhorias constantes, a partir da experiência daqueles que o utilizam (NOGUEIRA, 2003).

Haycox, Bagust e Walley (1999) observaram ainda que:

A implantação de procedimentos protocolados é complexa, porque invariavelmente vai refletir o conflito de poder entre as várias partes interessadas: os defensores da idéia, os fornecedores de medicamentos que estão interessados nesta fatia de mercado e as autoridades que estão interessadas em promover um incremento da qualidade ao mesmo tempo em que buscam reduzir os custos (HAYCOX; BAGUST; WALLEY, 1999, p. 392).

A busca pela qualidade não se limita apenas aos que estão envolvidos com a aplicação dos procedimentos padronizados. Ela deve ser uma meta que perpassa por todos os escalões do hospital, indo desde a mais alta administração até o mais simples dos funcionários. Nesse processo é esperado que aconteçam resistências por parte de todos os que estão envolvidos, principalmente entre um dos que é considerado uma peça fundamental nesse contexto: o médico (HAYCOX; BAGUST; WALLEY,1999; NOGUEIRA, 2003; GROL et al.,1998).

Tradicionalmente, a formação do médico é fortemente orientada para o trabalho individual. Isso faz com que, em 
geral, o médico se sinta totalmente responsável pela forma de conduzir o seu trabalho e pelo seu desfecho. Além disso, a formação eminentemente acadêmica do médico em geral não leva em conta uma preocupação bastante comum na área administrativa: o custo. O objetivo do médico é promover o bem-estar do paciente a qualquer preço. No entanto, devido ao aumento de complexidade das práticas médicas, ao grande arsenal técnico disponível e ao aumento progressivo dos custos, cada vez mais o médico tem precisado compartilhar com outros a responsabilidade global pelo cuidado com o paciente. Começam a surgir sistemas de controles nos quais o médico precisa explicar a adoção de procedimentos de alto custo em detrimento de outros que talvez tivessem a mesma repercussão e um custo mais baixo (FELICÍSSIMO, 2000; NOGUEIRA, 2003; SEGURA, 1994).

O médico e os demais profissionais da saúde não trabalham isoladamente e, sim, integrados em uma rede complexa de ações e procedimentos. Por outro lado, os serviços de saúde dispõem de quantidade limitada de recursos, que devem ser distribuídos segundo critérios preestabelecidos. Ao consumir qualquer tipo de recurso, seja através do fornecimento de um medicamento ou de uma autorização de internação, impede-se a possibilidade de um consumo alternativo; assim, otimizar os benefícios dos recursos empregados torna-se, antes de tudo, um imperativo ético (FELICÍSSIMO, 2000; SEGURA, 1994).

Nesse sentido, Bittar (2000) entende que "em um setor como o da saúde, em que os recursos não são abundantes, a variável custo torna-se extremamente importante, pois somente a sua contenção permitirá atender o maior número de pessoas".

Na opinião de Falk (2001), a análise da distribuição dos recursos hospitalares é particularmente complexa devido à natureza do serviço prestado, que se torna personalizado devido ao tipo de paciente atendido. Os pacientes diferem por conta do gênero, idade ou devido à resistência física e emocional de cada um, apresentando custos variáveis diferentes com base na quantidade de serviços ou atividades necessárias para a recuperação total de sua saúde.

A construção de um sistema de custos hospitalares requer, segundo Martins (2000), o entendimento dos seguintes fatores:

a) a estrutura organizacional do hospital;

b) os procedimentos médicos;

c) o tipo de informação de custos hospitalares desejados.

O sistema deve corresponder à divisão organizacional da autoridade de maneira que os responsáveis pelos diversos "empreendimentos" sejam também responsáveis pelas prestações de contas dos custos incorridos em suas áreas. Deve também refletir a forma como os procedimentos médicos foram realizados, indicando quais foram os materiais utilizados ou que tipo de serviços foram necessários para conclusão final do diagnóstico.

Construir um sistema de custeio baseado nos procedimentos hospitalares, de acordo com Matos (2002), signi- fica compor o custo sob a unidade do paciente e abrange, portanto, todos os insumos utilizados no fluxo percorrido por ele ao longo das diversas unidades funcionais de prestação de serviços existentes no hospital.

Para Matos (2002), "a metodologia de apropriação de custos dos procedimentos hospitalares propicia inegáveis benefícios ao processo de gestão" (MATOS, 2002, p. 197), destacando-se:

a) permite o agrupamento dos procedimentos hospitalares por especialidades médicas, facilitando a análise de resultados do hospital;

b) facilita o controle dos custos e recursos utilizados;

c) permite realizar comparações com outras instituições, quanto à qualidade, custos e preço dos tratamentos;

d) consiste em uma referência para o estabelecimento de preços sob a metodologia dos pacotes.

A disseminação da metodologia de apropriação de custos de procedimentos hospitalares aliada ao controle dos gastos e à busca pela qualidade dos serviços prestados têm levado ao interesse na construção de parâmetros ou modelos de gestão fundamentados na conduta médica adequada para o tratamento do paciente. Uma das formas atualmente discutida pela comunidade médica e administrativa é a utilização de modelos de gestão por processos também conhecida por modelos de gestão por protocolos de atendimento médico (grifos dos autores).

Este artigo tem por objetivo analisar se a utilização de protocolos de atendimento pode promover um incremento da qualidade dos serviços médico-hospitalares via padronização de processos e, dessa forma, promover uma redução dos custos da cirurgia e da internação diretamente relacionados aos procedimentos padronizados.

Na seqüência serão apresentados os marcos teóricos norteadores deste estudo, os procedimentos metodológicos utilizados para a consecução do objetivo proposto e os resultados encontrados após o processo de análise dos dados.

\subsection{Modelo de gestão por processos: os protocolos de atendimento médicos}

A idéia de utilizar protocolos nasceu dentro da engenharia civil, que buscava o máximo de eficiência no uso dos recursos por meio da criação de modelos de procedimentos para serem utilizados nas construções. Posteriormente, esse raciocínio foi adaptado para a medicina, por meio da criação de padrões de tratamento para serem utilizados no trato com os pacientes. Em meados de 1974 . foram implantados os primeiros protocolos em unidades intensivas com o objetivo de padronizar o tratamento e se certificar de que nenhum detalhe importante seria omitido no diagnóstico do paciente. Contudo, somente ao fim de 1980, pressionados por mudanças que permeavam o mercado, os protocolos médicos foram de fato implementados (FIELD; LOHR, 1990; LIN et al., 2001; NOGUEIRA, 2003). 
De acordo com Lin et al. (2001), inicialmente os protocolos foram desenvolvidos para serem utilizados por enfermeiras e outros profissionais (excetuando os médicos) que estão diretamente relacionados ao cuidado com o paciente. Contudo, com o crescimento da necessidade da medicina eficiente, os médicos também entraram no processo de uso dos protocolos. A partir daí, diferenças que porventura pudessem existir entre as formas de agir apresentadas pelos médicos eram dissolvidas e unificadas em torno de um "procedimento padrão", de forma que todos os pacientes tivessem praticamente as mesmas intervenções, independente do profissional que o atendesse.

Os protocolos representam uma seqüência ótima de procedimentos, efetuados no tempo certo, executado por médicos, enfermeiros e outros profissionais de saúde para um determinado diagnóstico ou procedimento. Uma das características mais importantes dos protocolos é a de que ele permite uma visão geral de cada procedimento que será realizado no paciente, com os respectivos resultados esperados, que podem ser monitorados por qualquer pessoa que esteja responsável pelo paciente, incluindo ele próprio (ARCHER, 1997; FIELD; LOHR, 1990; HUNTER; FAIRFIELD, 1997; MARTín et al., 2002; ORTON, 1994).

Para Field e Lohr (1990), os protocolos contêm todas as atividades que serão realizadas nos pacientes que apresentem determinadas patologias em seus aspectos de promoção, prevenção, diagnóstico, tratamento, reabilitação e cuidados crônicos. Correlacionam um sistema de avaliação de processos e resultados, assim como o grau de sua utilização. Englobam, portanto, desde as atividades puramente clínicas até as de promoção de saúde e prevenção da enfermidade, junto com os aspectos de avaliação.

Horn, Sharkey e Limmasch (1997) ensinam que em vez de enfocar apenas os procedimentos médicos, os protocolos de atendimento também analisam o conteúdo e o tempo despendido em cada etapa do processo de tratamento, para determinar como obter um melhor resultado com um mínimo de custo durante todo o trato com o paciente.

Na opinião de Archer (1997), o treinamento fornecido a todos os profissionais que lidam com o paciente (inclusive médicos e residentes) pode ser considerado o melhor caminho para promover a implantação dos protocolos e, uma vez implantados, devem sempre ser pesquisadas meIhorias no sentido de aumentar ainda mais sua eficiência.

Para Martín, Rieira e García (2002), os protocolos médicos possuem os seguintes objetivos:

a) melhorar a qualidade da prática clínica: a reflexão sobre a prática clínica e a incorporação da evidência científica disponível assegura uma melhora na qualidade global do processo. Esse é um objetivo desejável tanto para pacientes como para profissionais e gestores do serviço de saúde.

b) orientar o processo de tomada de decisões clínicas: os protocolos devem se converter em um instrumento de ajuda no processo de decisões ao incorporar uma seqüência sistematizada de atividades. Trata-se de um objetivo que beneficia tanto os pa- cientes como os profissionais de saúde, uma vez que pretende evitar variações inapropriadas da prática médica e que devem ser minimizadas em benefício do próprio paciente.

c) proporcionar a utilização adequada das tecnologias médicas: à medida que o uso das tecnologias médicas torna-se excessivo, expõe-se o paciente a procedimentos diagnósticos e terapêuticos desnecessários, e gastam-se mais recursos. Os protocolos relacionam a requisição de exames com a evidência dos benefícios obtidos com o seu uso.

d) facilitar a defesa médica em caso de eventuais litígios: os serviços de saúde são um campo no qual ocorrem muitos processos judiciais derivados de práticas errôneas por parte dos profissionais. A existência de um padrão "homogêneo" de conduta pode facilitar a defesa do profissional de saúde em caso de um eventual problema nessa área.

Lobach e Underwood (1998) afirmam que o conteúdo dos protocolos de atendimento pode ser estruturado em vários formatos de modo a facilitar a tomada de decisões. Alguns desses formatos incluem mapa de cuidados críticos, escala (benchmarks) de performance clínica, dentre outros. A escolha do formato depende da proposta do protocolo, da intenção do usuário, da forma de cuidados prestados, bem como da qualidade e da transparência da evidência científica que se deseja alcançar.

No entanto, sua aplicação não é tão fácil. Para Grol et al. (1998), a implantação de protocolos exige envolvimento multidisciplinar, profissionais competentes tanto da área médica como da administrativa, grandes investimentos em pesquisas, um bom sistema de informações e, acima de tudo, muito estudo e experiência.

Em seus estudos, esses pesquisadores concluíram que os protocolos desenvolvidos devem ser compatíveis com os valores existentes no grupo, rechaçando a idéia de procedimentos implantados de forma autoritária e indiscriminada. Os protocolos não devem demandar muitas alterações nas práticas atuais e devem ser definidos com precisão, por meio de orientações específicas para ações e decisões em diferentes casos.

\section{$1.3 \mathrm{O}$ uso dos protocolos de atendimento médico como uma ferramenta para o incremento da qualidade e controle dos custos hospitalares}

Para Shekelle et al. (1999), o uso dos protocolos auxilia os médicos na escolha da melhor intervenção a ser utilizada de acordo com a complexidade da patologia apresentada. Essa metodologia avalia não apenas a variação dos custos, a média de permanência e o grau de mortalidade decorrente de possíveis intervenções médicas, mas também pode mensurar a morbidade decorrente de mudanças nos sintomas e sinais fisiológicos dos pacientes. O objetivo é determinar as melhores práticas durante todo o trata- 
mento e identificar as informações críticas que sirvam de suporte clínico para a tomada de decisões.

De acordo com a Sociedade Brasileira de Cardiologia (2002), a utilização de protocolos médicos no Brasil, assim como em muitos outros países, está sendo amplamente divulgada e apoiada tanto nos hospitais públicos como nos privados. A literatura aponta duas correntes: uma se posicionando a favor, alegando que através da padronização dos processos, os protocolos podem promover um incremento da qualidade e uma redução dos custos (ARCHER, 1997; BUGLIOLI; ORTÚN, 2000; CAPUANO, 1995; FIELD; LOHR, 1990; FREEMAN; PER, 1997; GIFFIN; GIFFIN, 1994; GRIMSHAW; RUSSELL, 1993; HORN et al., 1997; LIN et al., 2001; LOBACH; UNDERWOOD, 1998; MARTíN et al., 2002; SHEKELLE et al., 1999; VIGIL et al., 1999; WORRAL; CHAULK, 1997) e outra que é contra a sua adoção, alegando fatores éticos, questionando sua real eficiência e apontando essa prática como sendo uma das causas do engessamento da medicina (FEDER et al., 1999; GROL et al., 1998; HAYCOX; BAGUST; WALLEY, 1999; JONES et al., 2003; WOOLF et al., 1999).

No entanto, o que se percebe nos estudos publicados, é que as experiências de utilização dos protocolos foram realizadas em hospitais que tinham uma situação financeira bem equilibrada, que apresentavam uma maturidade organizacional e que estavam situados em regiões geográficas que apresentavam uma boa situação social econômica. Porém, o que se deseja verificar, é até onde pode ser sentida a eficiência dos protocolos em hospitais que não possuam todas essas características reunidas. Por esse motivo, escolheu-se como centro do debate os Hospitais Universitários. De acordo com Lagioia (2002), grande parte dessas instituições apresenta orçamentos restritos e aqueles que estão situados em regiões geográficas menos favorecidas sob o aspecto econômico e social, encontram grandes dificuldades em realizar sua missão de ensino-pesquisa-extensão porque possuem sua estrutura sobrecarregada de atendimentos. Dessa forma, esses hospitais se encontram hoje diante de um paradoxo: como desempenhar eficientemente sua missão com recursos escassos.

Na seqüência, segue a apresentação do caso estudado neste artigo, que é a Clínica de Internação da Unidade de Ortopedia e Traumatologia do Hospital das Clínicas da Universidade Federal de Pernambuco (UOT/HC/UFPE).

\section{APRESENTAÇÃO DA UNIDADE DE ANÁLISE: CLÍNICA DE INTERNAÇÃO DA UNIDADE DE ORTOPEDIA E TRAUMATOLOGIA DO HOSPITAL DAS CLINNICAS DA UNIVERSIDADE FEDERAL DE PERNAMBUCO}

A razão da escolha da Clínica de Internação da UOT/ HC/UFPE em detrimento de outras existentes no hospital foi devida ao fato de que o médico responsável demonstrou grande interesse em participar do estudo e, principalmente, pela concordância dele e de sua equipe em participarem de todas as etapas prescritas e de fornecerem todo o material bibliográfico necessário. A escolha do procedimento a ser protocolado foi realizada em função das seguintes características:

a) alta prevalência;

b) custos elevados;

c) facilidade de abordar o processo.

Nesse sentido, foi realizada uma pesquisa no Serviço de Contas Médicas do HC/UFPE durante os meses de maio de 2003 a maio de 2004 a fim de serem verificados quais eram os procedimentos de traumatologia que se enquadravam nessa condição. Os resultados alcançados foram demonstrados no Quadro 10 :

Tendo em vista os resultados apresentados, verificou-se que os procedimentos relacionados com a artroplastia total do joelho foram não apenas os mais freqüentes, se não que eles superavam em muito o segundo colocado na relação. Além disso, a artroplastia corresponde a um dos procedimentos mais onerosos da traumatologia devido ao tipo de material que é utilizado. Por essa confluência de motivos e também seguindo a orientação do médico-chefe responsável pela Unidade, decidiu-se implantar os protocolos nesses procedimentos.

A palavra artroplastia deriva do grego arthron, que quer dizer articulação e plastos, que quer dizer modelado, ou

\begin{tabular}{|l|c|}
\hline Procedimentos ortopédicos mais freqüentes & Quantidade de ocorrência \\
\hline Artroplastia total do joelho & 38 \\
\hline Ressecção simples de tumor ósseo & 18 \\
\hline Amputação de perna & 17 \\
\hline Abertura de bainha tendinosa & 14 \\
\hline Tratamento cirúrgico do pé torto congênito & 14 \\
\hline Artroplastia de quadril & 13 \\
\hline
\end{tabular}

Fonte: Serviços de Contas Médicas HC/UFPE

Quadro 1 Demonstração dos procedimentos ortopédicos mais freqüentes durante os meses de maio de 2003 a maio de 2004 no HC/UFPE 
seja, significa o procedimento de reconstrução das articulações, feitas através da implantação de próteses.

As primeiras próteses apareceram em 1950, tendo a cabeça e haste femorais fixas por pressão ao canal femoral. As próteses modernas tiveram início na Inglaterra com Sir. Jonh Charnley em 1968, substituindo a cabeça, colo femoral e acetábulos fixados ao osso por polimetilmetacrilato, e por Guepar substituindo o joelho por componentes que formavam uma dobradiça, também com seus componentes fixados por polimetilmetacrilato (LIMA; FERREIRA, 2002).

As artroplastias constituem-se hoje como a solução para a incapacidade gerada pela artrose de múltiplas articulações nos pacientes de qualquer idade ou de articulação única nos idosos.

\section{PROCEDIMENTOS METODOLÓGICOS}

A estratégia de pesquisa utilizada consistiu na realização de quase experimento. Dentre os vários modelos existentes, optou-se pela escolha de um proposto por Campbell e Stanley (1979), conhecido por "séries temporais". A essência do delineamento da série temporal consiste na presença de um processo periódico de medidas com a introdução de uma variável experimental no processo, cujos resultados são avaliados por uma descontinuidade nas medidas registradas.

Os sujeitos experimentais da pesquisa corresponderam aos pacientes que foram submetidos às cirurgias de artroplastias de joelho no HC/UFPE. A variável experimental testada foi a implantação de protocolos de atendimentos médicos.

A unidade de análise na qual foi realizado o quase experimento foi a Clínica de Internação da Unidade de Ortopedia e Traumatologia do Hospital das Clínicas da UFPE (UOT/HC/UFPE).

A série temporal foi dividida em dois períodos distintos.

No $1^{\circ}$ período todos os pacientes foram atendidos normalmente, ou seja, sem a adoção dos protocolos. Esse período correspondeu a todo o ano de 2004 e aos meses de janeiro a junho de 2005 totalizando 18 meses. Os dados referentes a todo o ano de 2004 foram comparados com os do primeiro semestre de 2005 para que se pudesse verificar se havia uma homogeneidade de informações no período em análise e pudesse ser construída uma base confiável de comparação.

$\mathrm{O} 2^{\circ}$ período correspondeu aos meses de agosto a dezembro de 2005. Nessa fase todos os procedimentos foram realizados de acordo com o que foi estabelecido nos protocolos médicos. Ao final do $2^{\circ}$ período, foi realizada uma comparação com os dados coletados antes da adoção dos protocolos a fim de que pudessem ser retiradas as análises conclusivas sobre o processo.
O fato do $2^{\circ}$ período da pesquisa ser composto de 5 meses contra 18 referentes ao $1^{\circ}$ período é considerado pela autora como sendo um fator limitante para as hipóteses testadas.

A coleta de dados foi realizada através do registro dos indicadores de qualidade, dos custos diretos das cirurgias de artroplastias de joelho e dos custos diretos da internação referente a esses procedimentos.

Os protocolos iniciam-se a partir do momento em que o paciente se submete ao processo de internação para realizar a cirurgia de artroplastia de joelho e se estende até sua alta hospitalar. Neste artigo, serão apresentados os resultados do teste de três hipóteses sendo a primeira delas relacionada a indicadores de qualidade e as outras duas relacionadas a indicadores de custos.

Hipótese 1: A utilização de protocolos de atendimento na clínica de internação da Unidade de Ortopedia e Traumatologia do HC/UFPE melhora a qualidade dos serviços médico-hospitalares prestados aos pacientes que se submeteram ao procedimento de artroplastia total de joelho nessa Unidade.

Como o espectro da qualidade em saúde é muito abrangente, este estudo irá se deter nos indicadores de processo, mais especificamente falando, naqueles que podem ser gerados pelos protocolos médicos. Um dos objetivos da implantação dos protocolos é melhorar a qualidade do serviço prestado através da criação de rotinas otimizadas de práticas médicas e de atendimento ao paciente. Ressaltese, ainda, que, quando se fala em melhorar a qualidade de atendimento, refere-se à observação de um conjunto de indicadores pré-selecionados. Para o recorte deste artigo, serão apresentados quatro deles, os quais serão interpretados conjuntamente, conforme o Quadro 20.

A implantação dos protocolos visa à realização de internações programadas para que possa ser reduzido o

\begin{tabular}{|l|l|}
\hline Indicadores de qualidade & Situação esperada após a implantação dos protocolos \\
\hline 1.1 Dias de permanência total do paciente & Diminuição \\
\hline 1.2 Dias de espera na internação para realização da cirurgia & Diminuição \\
\hline 1.3 Dias de espera para alta pós-cirurgia & Diminuição \\
\hline 1.4 Tempo de duração da cirurgia & Diminuição \\
\hline
\end{tabular}

Fonte: Elaboração Própria

Quadro 2 Matriz dos indicadores de qualidade 
tempo global da permanência do paciente no leito hospitalar. O tempo de permanência antes da cirurgia pode ser reduzido através de internamentos realizados apenas mediante a marcação de cirurgias previamente agendadas, evitando-se o processo de se internar para garantir um leito e somente depois verificar um encaixe na programação cirúrgica. A permanência pós-cirúrgica também pode ser abreviada, através da construção de rotinas de altas hospitalares, estabelecendo-se um limite médio de tempo considerado ideal para a saúde do paciente. No que se refere ao indicador relacionado com o tempo de duração da cirurgia, é esperado que ocorra uma redução através do seguimento de rotinas estabelecidas previamente pela equipe.

Para a construção das duas hipóteses relacionadas aos custos, optou-se por utilizar a metodologia proposta por Falk (2001).

Falk (2001) divide os custos totais em custos diretos $\mathrm{e}$ indiretos (overhead). Os custos diretos são aqueles que estão clara e diretamente associados ao item em questão e, normalmente, estão sob o controle direto do responsável pelo serviço. Os demais custos podem ser considerados indiretos. Outro dado importante para a completa análise dos custos seria a introdução do custo médio, que representa o custo total do procedimento dividido pelo número de pacientes atendidos.

Falk (2001) salienta que o retorno sobre o tempo dedicado ao controle dos custos indiretos, em termos de recursos economizados, pode ser mínimo quando comparado com o retorno sobre o controle de seus próprios custos diretos. A identificação dos custos diretos e indiretos, portanto, pode ser o primeiro passo para um melhor controle de gastos no hospital ou na instituiçãa de saúde.

Os custos diretos em um hospital são em sua maioria gerados pelos próprios médicos (FALK, 2001; RUTLEDGE, 1996); portanto, espera-se que haja uma redução dos custos diretamente relacionados à cirurgia e à internação daqueles procedimentos que serão protocolados, uma vez que os protocolos serão criados e adotados por toda a equipe responsável pelo processo.

O registro dos custos diretos foi realizado pela autora da pesquisa, por meio da identificação de todos os gastos diretamente relacionados à cirurgia e à internação do paciente.

Os custos indiretos não possuem sua ocorrência relacionada com os procedimentos per si e por esse motivo não serão abordados neste estudo.

Hipótese 2: A utilização de protocolos de atendimento na clínica de internação da Unidade de Ortopedia e Traumatologia do HC/UFPE reduz os custos diretos das cirurgias de artroplastia total de joelho realizadas nessa Unidade.

Os custos diretos das cirurgias de artroplastia que são diretamente relacionados aos protocolos foram interpretados conforme o Quadro $3 \mathbf{0}$.

Os custos da mão-de-obra da equipe médica e auxiliar são compostos pelo somatório dos salários dos médicos residentes, do médico preceptor, do anestesista e dos auxiliares de enfermagem. O custo dos médicos residentes, do anestesista e dos auxiliares de enfermagem foi calculado em função do tempo em que eles permaneceram na sala de cirurgia. O médico preceptor é o médico responsável pela cirurgia que ensina aos residentes as técnicas cirúrgicas de colocação das próteses de joelho. Só existe um médico preceptor para prótese de joelho, de forma que todos os residentes aprendem as mesmas técnicas. O custo da mão-de-obra do médico preceptor foi calculado em função do tempo de duração da cirurgia. Durante todo o período estudado não houve nenhuma modificação na equipe médica e auxiliar participante do processo cirúrgico.

A rouparia é constituída dos seguintes itens: jaleco, calça, capote, pró-pé, máscara e gorro; os quais são distribuídos de acordo com a composição da equipe que participa da cirurgia. Os custos desses itens variam em função da quantidade de pessoas no bloco cirúrgico e foram calculados da seguinte maneira: a) o jaleco e a calça possuem apenas o custo da lavagem; b) o capote possui os custos da lavagem e esterilização; c) o pró-pé, a máscara, o gorro e as luvas são materiais descartáveis, cujo custo foi calculado em função da quantidade utilizada $x$ custo de aquisição.

Os materiais hospitalares da anestesia e da cirurgia correspondem, respectivamente, ao instrumental necessário para a realização de uma raquianestesia e de uma cirurgia de prótese do joelho. O custo desses materiais correspon-

\begin{tabular}{|l|c|}
\hline Composição dos custos diretos por cirurgia de artroplastia & Situação esperada após a implantação dos protocolos \\
\hline Mão-de-obra da equipe médica e auxiliar & Diminuição \\
\hline Rouparia da equipe médica e do paciente & Inalterado \\
\hline Materiais hospitalares e descartáveis utilizados na anestesia & Diminuição \\
\hline Medicamentos utilizados na anestesia & Inalterado \\
\hline Materiais hospitalares e descartáveis utilizados na cirurgia & Diminuição \\
\hline Gasoterapia & Diminuição \\
\hline Próteses & Inalterado \\
\hline Depreciação dos equipamentos & Diminuição \\
\hline Utilização da sala de recuperação pós-anestésica & Inalterado \\
\hline
\end{tabular}

Fonte: Elaboração Própria

Quadro 3 | Matriz dos custos diretos da cirurgia 
de ao processo de lavagem e esterilização dele. Os descartáveis correspondem aos demais materiais utilizados e possuem seu custo associado ao consumo.

Os medicamentos utilizados na anestesia são compostos pelas drogas, soros e soluções anti-sépticas utilizados com o paciente, cujo custo varia em função do consumo e de algumas peculiaridades, tais como, o tamanho do membro a ser operado, o fluxo sanguíneo do paciente etc.

A gasoterapia corresponde ao fornecimento de oxigênio ao paciente que se submete à cirurgia. O seu custo foi calculado em função do tempo de duração da cirurgia.

O custo da prótese corresponde ao valor despendido pelo HC/UFPE para adquirí-las.

A depreciação dos equipamentos utilizados na cirurgia foi calculada pelo método linear, em função do custo de aquisição dos equipamentos, utilizando-se uma taxa anual de $20 \%$. Por meio de uma proporção, encontrou-se a depreciação por dia e por hora. O custo final irá variar em função das horas de cirurgia.

Depois de realizada a cirurgia, o paciente é encaminhado para uma sala de recuperação que se situa dentro das dependências do bloco cirúrgico. Nessa sala, existem uma série de máquinas de monitoramento e uma equipe médica e de enfermagem. O custo dessa sala é determinado em função da depreciação das máquinas e da remuneração da equipe. O custo final varia em função do tempo que o paciente permanece na sala.

No período analisado, não houve variação dos salários dos membros da equipe médica e de enfermagem, bem como do preço de aquisição dos equipamentos, medicamentos, oxigênio e descartáveis utilizados no cálculo dos custos, tendo em vista que seus valores foram atualizados até o mês de janeiro de 2006.

Através da otimização dos processos advinda com a implantação dos protocolos, é esperado que ocorra uma redução dos custos relacionados com pessoal médico e auxiliar, com a depreciação dos equipamentos e com a gasoterapia, pois esses itens possuem seu custo calculado em função das horas trabalhadas e acredita-se que as cirurgias serão realizadas mais rapidamente. Também pode ocorrer uma redução dos custos dos materiais hospitalares e descartáveis da cirurgia e da anestesia, pois se acredita que será evitado o desperdício e a utilização desnecessária desses materiais.

Por outro lado, não é esperado que ocorra uma redução dos custos relacionados com os seguintes itens: a) utilização de medicamentos, tendo em vista que a dispensação é feita de acordo com o andamento da cirurgia, e depende do estado geral de saúde física e mental do paciente;

b) aquisição das próteses, uma vez que ela será utilizada na mesma quantidade com ou sem os protocolos;

c) utilização da rouparia, visto que não se espera que ocorra uma redução do número de integrantes da equipe médica;

d) utilização da sala de sala de recuperação pós-anestésica, tendo em vista que a permanência do paciente nessa sala está totalmente relacionada com a de seu estado geral de saúde e independe da adoção dos protocolos.

Hipótese 3: A utilização de protocolos de atendimento na clínica de internação da Unidade de Ortopedia e Traumatologia do HC/UFPE reduz os custos diretos da internação referentes às cirurgias de artroplastia total de joelho realizadas nessa Unidade.

Os custos diretos da internação referentes aos procedimentos de artroplastia de joelho foram interpretados conforme o Quadro $4 \mathbf{0}$.

Após ser finalizada a cirurgia, o paciente retorna para o leito onde fica internado até o seu completo restabelecimento. Durante esse período, ele recebe antibióticos e medicamentos para controle da dor e antinauseantes. O custo dos medicamentos dispensados varia de acordo com o estado geral de saúde do paciente e da quantidade de dias que este permanece internado. A rotina de dispensação de medicamentos para o paciente que se submeteu a uma cirurgia de prótese de joelho não foi alterada após a implantação dos protocolos.

Enquanto permanece internado, o paciente recebe a visita diária do médico residente plantonista, da enfermeira e do auxiliar de enfermagem. Estas visitas já eram padronizadas muito antes da existência dos protocolos e obedecem a uma rotina estabelecida pela equipe que trabalha na internação. No prontuário médico do paciente fica registrada a quantidade de visitas que foi realizada por cada um desses profissionais. O custo final dos cuidados médicos foi calculado em função do salário de cada um dos profissionais e da quantidade de visitas diárias realizadas.

O custo com a rouparia durante o período de internação do paciente corresponde a lavagem diária do lençol que cobre o leito.

\begin{tabular}{|l|c|}
\hline Composição dos custos diretos da internação & Situação esperada após a implantação dos protocolos \\
\hline Medicamentos & Diminuição \\
\hline Cuidados médicos & Diminuição \\
\hline Rouparia & Diminuição \\
\hline Alimentação & Diminuição \\
\hline Exames & Diminuição \\
\hline
\end{tabular}

Fonte: Elaboração Própria

Quadro 4 Matriz dos custos diretos da internação 
Todo paciente internado no HC/UFPE, bem como o seu acompanhante, recebe o café da manhã, o almoço, o jantar e 03 lanches. O custo da dieta irá variar em função da permanência do paciente no leito.

O paciente que se submete a uma intervenção cirúrgica deve realizar exames complementares a fim do acompanhamento do seu estado geral de saúde. O custo final dos exames varia em função das solicitações realizadas.

Os protocolos visam a obter internações programadas, evitando-se permanências desnecessárias. Por esse moti- vo, após a implantação dessa ferramenta, espera-se que haja uma redução de todos os custos diretos relacionados com a internação do paciente, uma vez que todos eles variam em função da ocupação do leito hospitalar.

Os dados coletados para o teste das hipóteses foram avaliados antes e após a implantação dos protocolos de duas maneiras: através da análise de tendências e através da análise de variância (ANOVA). A hipótese será aceita sempre que o $\mathrm{F}$ calculado for maior que o $\mathrm{F}$ tabelado (ou crítico).

\section{RESULTADOS ENCONTRADOS}

Durante os anos de 2004 e 2005 foram estudados 89 pacientes que se submeteram ao procedimento de artroplastia de joelho. Nesse período, foram coletados os dados referentes aos custos da cirurgia e da internação bem como aos indicadores de qualidade concernentes a esse procedimento. Os resultados obtidos foram dispostos na forma de médias mensais, conforme mostram os Gráficos 1 e 20 e a Tabela 10.

A análise dos dados dispostos no Gráfico 1 evidenciaram que, no período anterior à implantação dos protocolos, havia uma tendência de crescimento da permanência total do paciente no leito hospitalar. Essa tendência é revertida a partir do mês de agosto/05, quando a equação da reta apresenta uma inclinação decrescente $(0,1099 x$ antes dos protocolos contra $-0,3 x$ após os protocolos), refletindo o início do atendimento baseado em procedimentos padronizados.

A análise dos indicadores expostos na Tabela 1 mostrou que o tempo médio de permanência total do paciente era de aproximadamente 12 dias, quando cerca de 4 dias eram gastos esperando pela marcação da cirurgia e 7 dias eram destinados ao pós- operatório (não incluído o dia da realização da cirurgia). Após a implantação dos protocolos, a permanência total observada foi de cerca de 5 dias, sendo que desses, 1

\section{Dias de Permanência}

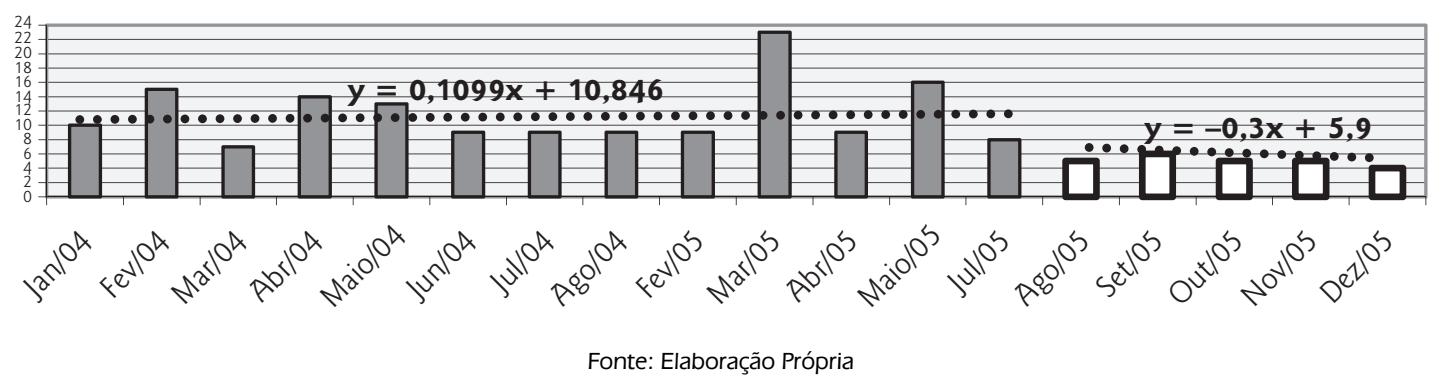

Gráfico 1 Análise da permanência média do paciente no leito (em dias)

Tempo de duração da cirurgia (min.)

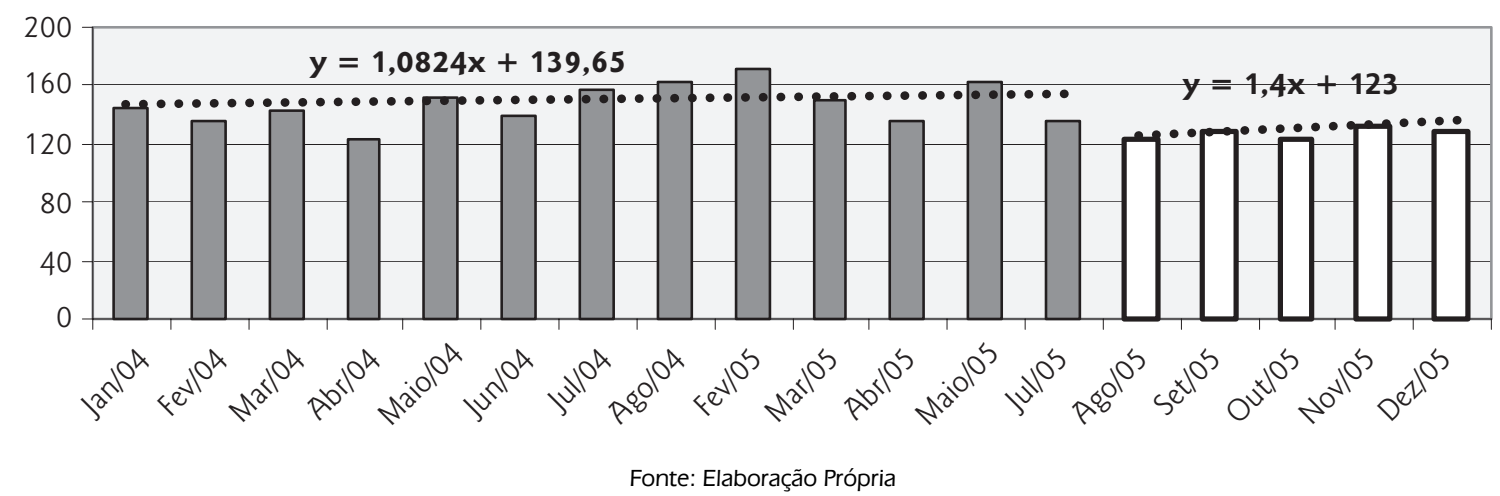

Gráfico 2 Análise do tempo médio de duração da cirurgia (em minutos) 
Tabela 1 || Resumo da análise da variância (ANOVA) dos indicadores de qualidade

\begin{tabular}{l|c|c|c|c|c|c} 
& $\begin{array}{c}\text { Média } \\
\text { do grupo } \\
\text { antes dos } \\
\text { protocolos }\end{array}$ & $\begin{array}{c}\text { Variância } \\
\text { do grupo } \\
\text { antes dos } \\
\text { protocolos }\end{array}$ & $\begin{array}{c}\text { Média do } \\
\text { grupo após } \\
\text { protocolos }\end{array}$ & $\begin{array}{c}\text { Variância } \\
\text { do grupo } \\
\text { após } \\
\text { protocolos }\end{array}$ & F calculado & F crítico \\
\hline $\begin{array}{l}\text { Dias de permanência } \\
\text { total (média) }\end{array}$ & 12 & 19,92 & 5 & 0,50 & 10,48854854 & 4,493998063 \\
\hline $\begin{array}{l}\text { Dias de permanência } \\
\text { antes da cirurgia (média) }\end{array}$ & 4 & 4,06 & 1 & 0,30 & 9,775862069 & 4,493998063 \\
\hline $\begin{array}{l}\text { Dias de permanência } \\
\text { após cirurgia (média) }\end{array}$ & 7 & 14,94 & 3 & 0,20 & 3,413965324 & 4,493998063 \\
\hline $\begin{array}{l}\text { Tempo de duração da } \\
\text { cirurgia (minutos) }\end{array}$ & 147 & 192,53 & 127 & 18,20 & 9,727750449 & 4,493998063 \\
\hline
\end{tabular}

Fonte: Elaboração Própria

dia foi destinado à espera da cirurgia e 3 dias foram dedicados ao pós-operatório. A análise da variância (ANOVA) apontou que ocorreu uma variação significativa das médias no que se refere à permanência antes da cirurgia, no entanto, a variação da permanência pós-cirurgia não foi significativa entre os dois períodos estudados. Porém, ao se observarem as variações das médias da permanência total, verifica-se que houve uma mudança significativa após a introdução dos protocolos. Percebe-se, assim, que um dos maiores problemas observados na UOT/HC/UFPE, ou seja, a elevada permanência, pode ser controlada revertendo em benefícios sobretudo para os pacientes atendidos, mas também para a equipe de profissionais envolvida e para a própria instituição hospitalar. Sabe-se que uma permanência menor reduz infecções hospitalares, promove o bem estar psicólogico dos pacientes (pelo retorno precoce para casa) e permite que mais pessoais possam acessar o Sistema de Saúde Público.

Através da análise dos dados dispostos no Gráfico 2, verificou-se que, no período posterior à implantação dos protocolos mesmos, houve uma queda inicial na tendência (123 minutos contra 139 minutos verificados no período anterior aos protocolos), seguida por uma inclinação mais acentuada que a existente no período anterior ( $1,4 \mathrm{x}$ após os protocolos contra $1,0824 x$ verificados antes dos protocolos).

De fato, após a implantação dos protocolos, ocorreu uma redução média de aproximadamente 20 minutos na duração da cirurgia, sendo essa variação significativa entre os dois períodos estudados (ver dados dispostos na Tabela 2). A médio e a longo prazos, contudo, esse tempo pode subir se não houver um rígido controle por parte dos intergrantes da equipe médica, tendo em vista a tendência constatada de crescimento progressivo.

Dessa maneira, pode-se concluir que a hipótese $\mathbf{1}$ foi confirmada para todos os indicadores de qualidade selecionados. Essa confirmação irá trazer reflexos diretos nas hipóteses 2 e 3, conforme se verá no Gráfico 30 e na Tabela 20.

A análise dos dados evidenciados no Gráfico 3 mostrou que a tendência dos custos totais da cirurgia acompanhou a tendência do tempo de duração da cirurgia, apresentando uma queda inicial $(R \$ 3.156,10$ antes dos protocolos contra $R \$ 3.122,70)$ seguida por uma tendência de crescimento superior ao verificado antes da implantação dos protocolos $(8,361 x$ contra $2,0647 x)$.

\section{Custo diretos variáveis da cirurgia}

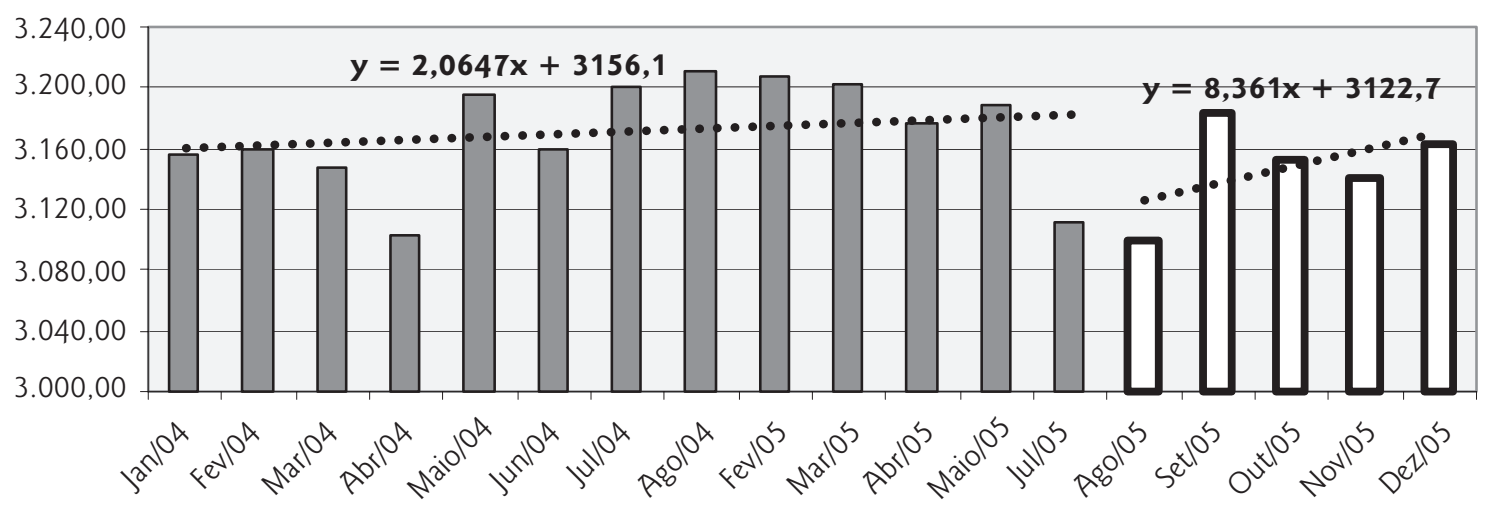

Fonte: Elaboração Própria

Gráfico 3 - Análise dos custos médios diretos da cirurgia (em R\$) 
Tabela 2 Resumo da análise da variância (ANOVA) dos custos diretos da cirurgia

\begin{tabular}{l|c|c|c|c|c|c} 
& $\begin{array}{c}\text { Média } \\
\text { grupo } \\
\text { antes dos } \\
\text { protocolos }\end{array}$ & $\begin{array}{c}\text { Variância } \\
\text { grupo } \\
\text { antes dos } \\
\text { protocolos }\end{array}$ & $\begin{array}{c}\text { Média } \\
\text { grupo } \\
\text { após } \\
\text { protocolos } \\
\text { Custo da mão-de-obra } \\
\text { equipe médica e auxiliar }\end{array}$ & $\begin{array}{c}\text { Variância } \\
\text { grupo } \\
\text { após } \\
\text { protocolos }\end{array}$ & F calculado & F crítico \\
\hline Custo da rouparia & 813,91 & 682,56 & 178,39 & 35,75 & 8,7482116 & 4,493998 \\
\hline $\begin{array}{l}\text { Custo dos descartáveis } \\
\text { usados na anestesia }\end{array}$ & 67,98 & 39,54 & 68,92 & 18,59 & 0,0936471 & 4,493998 \\
\hline $\begin{array}{l}\text { Custo dos medicamentos } \\
\text { usados na anestesia }\end{array}$ & 25,15 & 9,56 & 25,68 & 16,47 & 0,0871568 & 4,493998 \\
\hline $\begin{array}{l}\text { Custo dos descartáveis } \\
\text { usados na cirurgia }\end{array}$ & 411,93 & 391,93 & 434,23 & 910,27 & 3,4434698 & 4,493998 \\
\hline Custo da gasoterapia & 0,71 & 0,00 & 0,61 & 0,00 & 10,323705 & 4,493998 \\
\hline Custo da depreciação & 35,29 & 11,04 & 30,32 & 1,40 & 10,323705 & 4,493998 \\
\hline $\begin{array}{l}\text { Custo da recuperação } \\
\text { pós-anestésica }\end{array}$ & 52,85 & 268,48 & 52,31 & 54,57 & 0,0049856 & 4,493998 \\
\hline Custo direto total da cirurgia & $3.170,60$ & $1.250,69$ & $3.147,80$ & 954,43 & 1,5951446 & 4,493998
\end{tabular}

Fonte: Elaboração Própria

Os dados contidos na Tabela 2 permitem uma visualização dos itens de custo que sofreram alterações após a implantação dos protocolos. Desta maneira, pode-se observar que a média dos custos diretos totais da cirurgia no período posterior aos protocolos foi ligeiramente menor que no período anterior ( $\mathrm{R} \$ 3.147,80$ contra $\mathrm{R} \$ 3.170,60)$. No entanto, verificando-se item por item, observou-se que nem todos os componentes integrantes do custo se comportaram da mesma maneira.

Através da análise comparativa das médias, pode-se verificar que os custos diretos relacionados à mão-de-obra da equipe médica e auxiliar, após o processo de implantação dos protocolos caíram como previsto (redução média de $R \$ 213,91$ para $R \$ 178,39$, ou aproximadamente $17 \%$ ), devido à redução de cerca de 20 minutos do tempo médio de duração da cirurgia. No entanto, como esses custos possuem pouca representatividade na composição final, a variação não foi percebida a ponto de alterar significativamente a média dos custos totais.

Os gastos com a gasoterapia e com a depreciação dos equipamentos, também como já era esperado, mostraramse sensíveis aos protocolos e tiveram seus valores reduzidos, uma vez que esses custos estão relacionados diretamente com o tempo de duração da cirurgia. Os custos da gasoterapia caíram em média de $R \$ 0,71$ para $R \$ 0,61$ e os custos da depreciação apresentaram queda de $R \$ 35,29$ para $R \$ 30,32$ (uma redução em ambos os casos de cerca de $14 \%$ ). Porém como esses valores são pouco representativos, a queda ocorrida não alterou substancialmente 0 resultado final.

Um item que mostrou um resultado distinto do esperado foram os custos relacionados com materiais hospitalares e descartáveis utilizados tanto na anestesia quanto na cirurgia. A idéia inicial era de que após a implantação dos protocolos, haveria uma redução desses custos devido a uma melhor utilização desses materiais, evitando-se o desperdício. Porém, o que foi constatado na prática foi outra realidade, ou seja, os valores se mantiveram praticamente os mesmos. A média dos custos dos descartáveis da anestesia variou de $R \$ 67,98$ para $R \$ 68,92$ e a dos descartáveis da cirurgia de $R \$ 411,93$ para $R \$ 434,23$, (variando menos de 1\% e 5\%, respectivamente). Na verdade, os gastos com os descartáveis e materiais hospitalares já estavam ajustados às necessidades da cirurgia. Não foi constatado o uso de materiais desnecessários ou em quantidade indevida. Muito pelo contrário, sempre se buscou uma utilização racional desses recursos.

Os custos dos medicamentos utilizados na anestesia, como já era previsto, mantiveram-se constantes tendo em vista que cada paciente recebe uma quantidade de drogas compatível com a sua necessidade (variação de $R \$ 25,15$ para $R \$ 25,68$, ou seja, em torno de $2 \%$ entre os dois períodos). Da mesma forma, os custos com a rouparia apresentaram pouca oscilação (variação de $R \$ 88,93$ para $R \$$ 83,50 , ou aproximadamente $6 \%$ ), devido à composição da equipe permanecer a mesma.

Os custos ligados com a sala de recuperação pós-anestésica, embora sejam diretamente relacionados com o processo cirúrgico, estão fora da abrangência dos protocolos, pois o tempo de permanência nessa sala irá depender do estado de saúde geral do paciente. Mesmo assim, realizouse o teste comparativo de médias e verificou-se que não houve alteração significativa entre os períodos estudados.

$O$ custo de aquisição das próteses ( $R \$ 2.273,85)$ se manteve inalterado tendo em vista que a sua utilização foi a mesma, independentemente dos protocolos. Por este motivo esse custo foi suprimido da apresentação dos resultados da Tabela 2. 
Dessa maneira, pode-se afirmar que a hipótese $\mathbf{2}$ não foi confirmada quando se trata da análise global dos custos relacionados à cirurgia. Na análise segmentada desses custos, a hipótese 2 foi confirmada para os itens relacionados com a mão-de-obra da equipe médica e auxiliar, com a gasoterapia e com a depreciação dos equipamentos, que tiveram seus valores reduzidos após a implantação dos protocolos. Por outro lado não se confirma a hipótese 2 para os custos relacionados com materiais e descartáveis utilizados na cirurgia e na anestesia.

A análise dos dados referente aos custos da internação apresentou modificações mais sensíveis que as verificadas nos custos da cirurgia. No Gráfico $4 \mathbf{0}$, percebe-se a formação de uma tendência descendente após o período de implantação dos protocolos devida, principalmente, à redução do tempo de permanência do paciente no leito de aproximadamente de 12 para 5 dias. A redução do tempo de permanência, por sua vez, reduziu os custos diretos da internação, uma vez que eles estão intrinsecamente relacionados.

Analisando-se os dados da Tabela $3 \mathbf{O}$, verificou-se que a média dos custos diretos da internação caíram de $\mathrm{R} \$$ 750,53 para $R \$ 335,43$ (cerca de $55 \%$ ). Os custos com os cuidados médicos também foram reduzidos de aproximadamente $\mathrm{R} \$ 281,25$ para $\mathrm{R} \$ 121,80$ (queda de $57 \%$ ). Da mesma forma, os custos com a rouparia baixaram de $R \$$ 19,88 para $\mathrm{R} \$ 8,67$ (redução média de 56\%).
No entanto, alguns elementos formadores desse custo mostraram uma posição contrária ao esperado. O teste de comparação das médias mensais dos custos dos medicamentos e dos serviços auxiliares de diagnóstico e tratamento (SADT) não mostrou uma alteração significativa no período anterior e no posterior à adoção dos protocolos. Ao que tudo indica, mesmo com a permanência reduzida, existem variações de custos nesses dois itens. De fato, ocorreram situações em que o paciente permaneceu internado por 4 ou 5 dias, mas, nesse período de tempo, houve a necessidade de dispensação de medicamentos especiais e mais caros, devido a sua condição de saúde (paciente diabético, hipertenso etc.). Raciocínio análogo pode ser aplicado às solicitações de exames, os quais também apresentaram oscilações em ambos períodos, dependendo da necessidade individual do paciente.

Ocorre que um paciente que necessite de medicamentos especiais, trará mais custos ao hospital se apresentar uma permanência de 7 ou 8 dias do que se passar apenas uma de apenas 4 dias. Em outras palavras, se o tempo de permanência do paciente no leito é menor, o gasto necessariamente será menor, conforme foi verificado na análise de tendência.

No processo de elaboração dos protocolos, uma das maiores preocupações que se teve foi a de criar rotinas que reduzissem essa permanência sem afetar a qualidade dos serviços prestados ao paciente, buscando equacionar da melhor maneira a relação custo $\mathrm{x}$ benefício para o hospital.

Custo diretos variáveis da internação

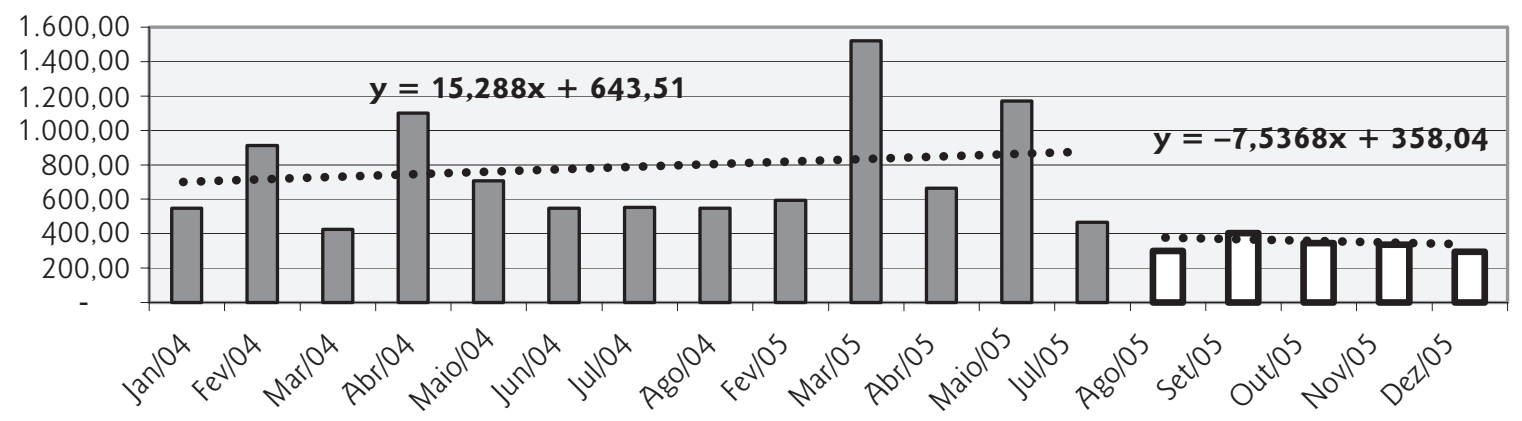

Fonte: Elaboração Própria

Gráfico 4 Análise dos custos médios diretos da internação (em R\$)

Tabela 3 Resumo da análise da variância (ANOVA) dos custos diretos da internação

\begin{tabular}{|c|c|c|c|c|c|c|}
\hline & $\begin{array}{c}\text { Média } \\
\text { grupo } \\
\text { antes dos } \\
\text { protocolos }\end{array}$ & $\begin{array}{l}\text { Variância } \\
\text { grupo } \\
\text { antes dos } \\
\text { protocolos }\end{array}$ & $\begin{array}{c}\text { Média } \\
\text { grupo } \\
\text { após } \\
\text { protocolos }\end{array}$ & $\begin{array}{l}\text { Variância } \\
\text { grupo } \\
\text { após } \\
\text { protocolos }\end{array}$ & F calculado & F crítico \\
\hline Custo dos medicamentos & 120,03 & $10.189,02$ & 64,49 & 234,84 & 1,4466758 & 4,493998 \\
\hline Custo dos cuidados médicos & 281,25 & $12.935,13$ & 121,80 & 285,87 & 9,3935864 & 4,493998 \\
\hline Custo da rouparia & 19,88 & 56,85 & 8,67 & 1,46 & 10,559185 & 4,493998 \\
\hline Custo da alimentação & 302,28 & $13.843,35$ & 126,68 & 424,76 & 10,615885 & 4,493998 \\
\hline Custo dos exames (SADT) & 27,09 & 317,64 & 13,79 & 16,97 & 2,635593 & 4,493998 \\
\hline Custo direto total da internação & 750,53 & $108.412,81$ & 335,43 & $1.898,01$ & 7,6080963 & 4,493998 \\
\hline
\end{tabular}


Por esse motivo, pode-se afirmar que, nesse caso, a hipótese 3 foi confirmada para o custo variável da inter- nação não apenas no contexto global, mas em todos os itens específicos.

\section{CONCLUSÕES}

Os resultados deste estudo mostraram que a utilização de protocolos de atendimento na clínica de internação da Unidade de Ortopedia e Traumatologia do HC/UFPE provocou uma melhora em todos os indicadores de qualidade selecionados. No que concerne aos custos diretos relacionados com a cirurgia, verificou-se que não houve uma redução dos mesmos quando se observa os resultados de forma conjunta. Porém, os itens de custeio relacionados com a mão de obra da equipe médica e auxiliar, com a gasoterapia e com a depreciação dos medicamentos tiveram seus valores reduzidos após a implantação dos protocolos. Por outro lado, os custos diretos da internação tiveram seus valores reduzidos não apenas em todo o conjunto estudado, mas em cada um dos itens analisados.

A direção do hospital e o corpo clínico da UOT/HC/ UFPE reconhecem a validade dos resultados encontrados. Os protocolos implantados geraram um Manual de Conduta e Procedimentos, o qual se encontra em uso na referida Unidade.

Este artigo apontou para o fato de que mesmo em Hospital Universitário, com um orçamento restrito e pouco flexível, a implantação dos protocolos na Unidade de Ortopedia e Traumatologia do HC/UFPE conseguiu cumprir com a maioria dos objetivos que foram determinados, melhorando a qualidade do atendimento prestado e reduzindo os custos dos procedimentos hospitalares.

A forma pela qual uma mudança é implantada em uma organização é um dos maiores fatores responsáveis para que ela seja aceita e gere resultados. No caso em questão, a participação de todos os envolvidos, as reuniões para esclarecimentos e o acompanhamento sistemático dos resultados que vinham sendo alcançados foram decisivos para que este trabalho alcançasse sua finalidade.

Todavia, deve-se ressaltar que os resultados encontrados nesta pesquisa limitam-se ao âmbito do caso estudado. Não se pode afirmar que outras unidades do HC/UFPE, ou então, que uma unidade de ortopedia e traumatologia de um hospital distinto venha a alcançar os mesmos resultados. Estudos adicionais seriam necessários para se verificar a possibilidade de generalização.

Como sugestão de outras pesquisas futuras, recomenda-se ainda a investigação de outros fatores que podem ter influenciado nesse processo, tais como: os fatores sociológicos, os organizacionais, os comportamentais e os motivacionais. Tais variáveis não foram abordadas neste estudo em função de a autora ter escolhido a vertente mais quantitativa, relacionada aos custos e aos indicadores de qualidade gerados pelos próprios protocolos. No entanto, outras áreas podem debruçar-se sobre este mesmo assunto e apresentar contribuições relevantes para o estudo. A pluralidade de abordagens enriquece sobremaneira as conclusões que podem ser obtidas sobre o tema, uma vez que, cada especialista, ao olhar para o mesmo problema, identifica determinados fatores relacionados com a sua área e oferece uma explicação complementar para o entendimento do processo.

\section{Referências}

ANDRADE,M. Custos na atividade hospitalar. Temas contábeis em destaque. Conselho Regional do Estado de São Paulo. Coordenação José Barbosa da Silva Júnior. São Paulo: Atlas, 2000. (Coleção seminários CRC/SP/IBRACON).

ARCHER, S. Implementation of a clinical pathway decreases length of stay and hospital charges for patient undergoing total colectomy and ileal pouch/anal anastomosis. In: ANNUAL MEETING OF THE CENTRAL SURGICAL ASSOCIATION, 54., 1997, Chicago. Department of surgery, University of Cincinnati Medical Center, Ohio.

BEULKE, R.; BERTÓ, D. J. Gestão de custos e resultado na saúde: hospitais, clínicas, laboratórios e congêneres. 1. ed. São Paulo: Saraiva, 1997. BITTAR, O. J. N. V. Gestão de processos e certificação para qualidade em saúde. Revista da Associação Médica Brasileira, São Paulo, v. 46, n. 1, jan./mar. 2000.

BUGLIOLI, M.; ORTUN, V. Decisión clínica: como entenderla y mejorarla. Barcelona: Springer, 2000.

CAMPBELL, D. T.; STANLEY, J. C. Delineamentos experimentais e quase-experimentais. São Paulo: EPU: USP, 1979, 138 p.

CAPUANO, T. A. Clinical pathways. Nursing Management, Chicago, v. 26, n. 1, p. 34-38, jan. 1995.

FALK, J. Gestão de custos para hospitais: conceitos, metodologias e aplicações. São Paulo: Atlas, 2001.

FEDER, G. et al. Using clinical guidelines. BMJ Publishing Group, v. 318, p. 728-730, Mar. 1999.

FELICÍSSIMO, A. Economia da saúde. Revista de administração em saúde, v. 2, n. 6, p. 13-16, 2000.

FIELD, M. J.; LOHR, K. N. Committee to advise the public health service on practice guidelines, Institute of Medicine, Clinical Practice Guidelines: Directory for a New Program. Washington, D. C., National Academy Press, 1990, p. 38, 
FREEMAN, G.; PER, H. What future for continuity of care in general practice? BMJ Publishing Group, v. 313, p. 1870-1873, 1997.

GIFFIN, M.; GIFFIN, R. B. Market memo: critical pathways produce tangible results. Health Care Strategic Management, Chicago, v. 12, n. 7 , p. 1-7, jul. 1994.

GRIMSHAW, J. M.; RUSSELL, I. T. Effect of clinical guideline on clinical medical practice: a systematic review of rigorous evaluations. Lancet, v. 342, p. 1317-1322,1993.

GROL, R. et al. Attributes of clinical guidelines that influence use of guidelines in general practice: observational study. BMJ Publishing Group, v. 317, p. 858-861, sept. 1998.

HAYCOX, A.; BAGUST, A.; WALLEY, T. Clinical guidelines: the hidden costs. BMJ Publishing Group, v. 318, p. 391-393, Feb. 1999.

HORN, S. D.; SHARKEY, S. S.; LIMMASCH, H. L. Clinical practice Improvement: a methodology to improve quality and decrease cost in health care. Oncology Issues, v. 12, n. 1, p. 16-20, 1997.

HUNTER, D.; FAIRFIELD, G. Disease management. BMJ, v. 315, p. 50-53, 1997.

JONES, J. W.; McCULLOUGH, B.; RICHMAN, B. W. The ethics of clinical pathwaysand cost control. Journal of Vascular Surgery, v. 37, n. 6, p. 1341-1342, June 2003

LAGIOIA, U. C. T. Estudo de novas fontes de financiamento nos hospitais universitários: o caso do Hospital das Clínicas da Universidade Federal de Pernambuco. 2002. 204 f. Dissertação (Mestrado em Administração) - Universidade Federal de Pernambuco, Recife.

LIMA, J.A.; FERREIRA, A. M. A. Relatório do serviço social no programa de mutirão de artroplastia de quadril e joelho no serviço de traumatoortopedia do HC/UFPE no período de outubro de 2000 a agosto de 2001. Universidade Federal de Pernambuco. Recife: Universidade Federal de Pernambuco, 2002 .

LIN, F. et al. Mining time dependency patterns in clinical pathways. International Journal of Medical Informatics, v. 62, n. 1, p. 11-25, June 2001.

LOBACH, D. F.; UNDERWOOD, H. R. Computer-based decision support systems for implementing clinical practice guidelines. Drug Benefit Trend, v. 10, n. 10, p. 48-53, 1998.

MARTÍN, E. G.; RIERA, J. M. C.; GARCÍA, F. I. Mejorando la gestión clínica: desarrollo e implantación de guías de práctica clínica. Barcelona: CHC Consultoria i Gestió, 2002.

MARTINS, D. Custos e orçamentos hospitalares. São Paulo: Atlas, 2000.

MATOS, A. J. Gestão de custos hospitalares: técnicas, análise e tomada de decisão. São Paulo: STS, 2002.

NOGUEIRA, L. C. L. Gerenciando pela qualidade total na saúde. Belo Horizonte: Editora de Desenvolvimento Gerencial, 2003

ORTON, P. Shared care. Lancet, v. 344, p. 1413-1415, 1994.

RUTLEDGE, V. R. Hospital physician alignment: a model for success. Oncology Issues v. 11, n. 6, p. 18-20, 1996. Association of Community Centers.

SEGURA, A. In: BADIA, X.; FORNS, R. J. Prólogo de evaluacion econômica de medicamentos un instrumento para la tomada de decisión en la practica clínica y la política sanitária. 5. ed. Luzán: 1994.

SHEKELLE, P. G. et al. Developing guidelines. BMJ Publishing Group, v. 318, p. 593-596, Feb. 1999.

VIGIL, B. M.; SIMON, L. J.; GLARIA, N. T. Estudio de la disminución de la mortalidad a partir de la protocolización y control de las enfermedades crónicas y sus factores de riesgo. In: CONGRESSO DE LA SOCIEDAD ESPAÑOLA DE MEDICINA FAMILIAR Y COMUNITARIA, 19., 1999, Tenerife. Anales... Tenerife, 1999.

WOOLF, S. H. et al. Clinical guidelines potential benefits, limitations, and harms of clinical guidelines. BMJ Publishing Group, v. 318, p. 527530, Feb. 1999.

WORRAL, G.; CHAULK, P. The effects of clinical practice guidelines on patients outcomes in primary care: a systematic review. Can. Med. Assoc. J., v. 156, p. 1705-1712, 1997.

\section{NOTA - Endereço dos autores}

Universidade Federal de Pernambuco Centro de Ciências Sociais Aplicadas Departamento de Ciências Contábeis Av. dos Funcionários, S/n, $1^{\circ}$ andar - Cidade Universitária Recife - PE

50740-580
Faculdade Boa Viagem Departamento de Ciências Administrativas R. Jean Emile Favre, 422 - Imbiribeira Recife - PE

$51200-060$ 